\title{
Lipoma of the Finger with Bone Erosion
}

\author{
Yasuyuki Kitagawa ${ }^{1,2}$, Kensuke Tamai ${ }^{1,2}$, Yong Kim ${ }^{1,2}$,

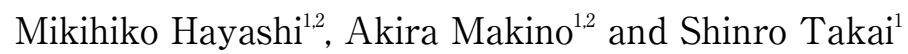 \\ ${ }^{1}$ Department of Restorative Medicine of Neuro-musculoskeretal System, Orthopaedic Surgery, \\ Graduate School of Medicine, Nippon Medical School \\ ${ }^{2}$ Department of Orthopaedic Surgery, Nippon Medical School Tama Nagayama Hospital
}

\begin{abstract}
Lipoma of the finger is rare, and there have been few case reports of lipoma causing bone erosion in the finger. We report on a patient with a long-standing lipoma that caused deep erosion of the distal phalanx of the index finger. A 38-year-old man presented with a painful mass of the left index finger. Anteroposterior radiographic images showed a radiolucent area in slightly distal to the center of the distal phalanx. Magnetic resonance imaging revealed a large soft-tissue mass with high signal intensities and slight irregularity on both T1- and T2weighted images which suggested lipoma. The patient underwent marginal excision of this tumor and curettage of the bone surface of the erosion. The pain resolved after surgery. The diagnosis of lipoma was confirmed with postoperative histological examination. Lipoma should be included in the differential diagnoses of a finger tumor, even if radiography shows bone erosion.
\end{abstract}

(J Nippon Med Sch 2012; 79: 307-311)

Key words: lipoma, hand, finger, bone change

\section{Introduction}

Lipoma is a common soft tissue tumor that can occur in any part of the body. However, lipoma rarely occurs in the finger and rarely causes bone erosion. There have been a few case reports of lipoma causing bone erosion in the finger ${ }^{1-3}$. We report on a patient with a long-standing lipoma that caused deep erosion of the distal phalanx of the index finger. Differential diagnoses for this lesion are discussed.

\section{Case Report}

A 38-year-old man presented with a mass of the left index finger. The mass had slowly enlarged over approximately 18 years and had been painful for the previous 2 years. There was no history of trauma to the hand. His medical history was unremarkable. On physical examination, a mass covered with pink, thin, taut skin was detected on the pulp of the index finger (Fig. 1). Superficial dilated veins were observed through the skin. The mass was painful, relatively firm, and immobile. The nail was elevated and convex, and the nail bed was pale. The range of

Correspondence to Yasuyuki Kitagawa, MD, Department of Orthopaedic Surgery, Nippon Medical School Tama Nagayama Hospital, 1-7-1 Nagayama, Tama, Tokyo 206-8512, Japan

E-mail: kitayasu@nms.ac.jp

Journal Website (http://www.nms.ac.jp/jnms/) 
motion of the distal interphalangeal joint was restricted $\left(0-30^{\circ}\right)$. No sensation disturbance was found in the finger.

Anteroposterior radiographic images showed a radiolucent area slightly distal of the center of the

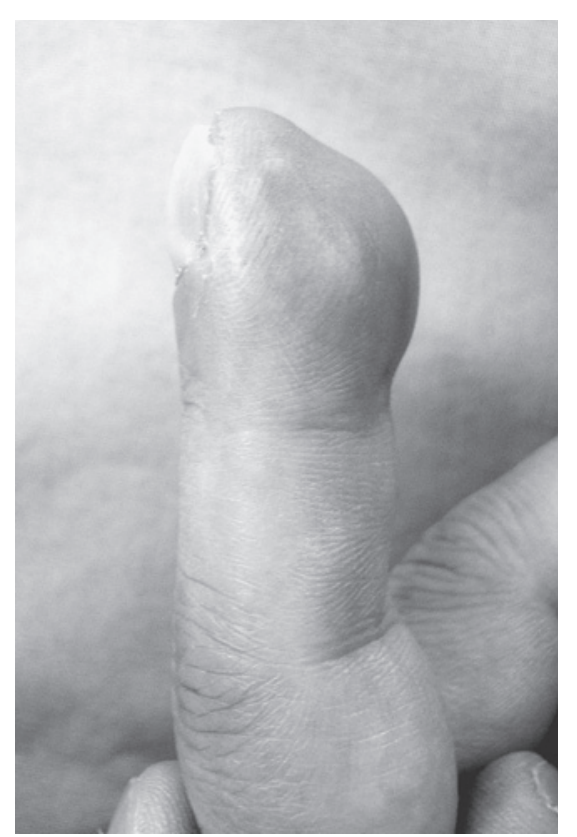

Fig. 1 A preoperative lateral view of the index finger. A mass on the pulp of the index finger with pink, thin, taut skin and elevated and convex nail. distal phalanx. This area was round and resembled an enlarged vessel foramen, although no vessel foramen of the distal phalanx was observed on the unaffected side (Fig. 2). On a lateral view, a deep, narrow bone erosion was observed to extend into the medullary space with a sclerotic peripheral margin. Magnetic resonance imaging revealed a large soft-tissue mass with high signal intensities and slight irregularity on both T1- and T2-weighted images which suggested lipoma (Fig. 3). The lesion was present on both the palmar and dorsal sides of the distal phalanx and extended proximally to the palmar soft tissue between the distal and middle finger creases. The lesion also extended into the medullary space through the palmar cortex, but whether it penetrated through the dorsal cortex was unclear. Fine-needle aspiration biopsy showed many mature perivascular adipocytes, which also suggested lipoma.

The patient underwent marginal excision of this tumor and curettage of the bone surface of the erosion. At surgery, a soft yellow mass was observed under the skin. The mass was lobular and extended into the bone through the palmar cortex of the distal phalanx but did not penetrate the dorsal cortex of the phalanx. The lesion also extended to

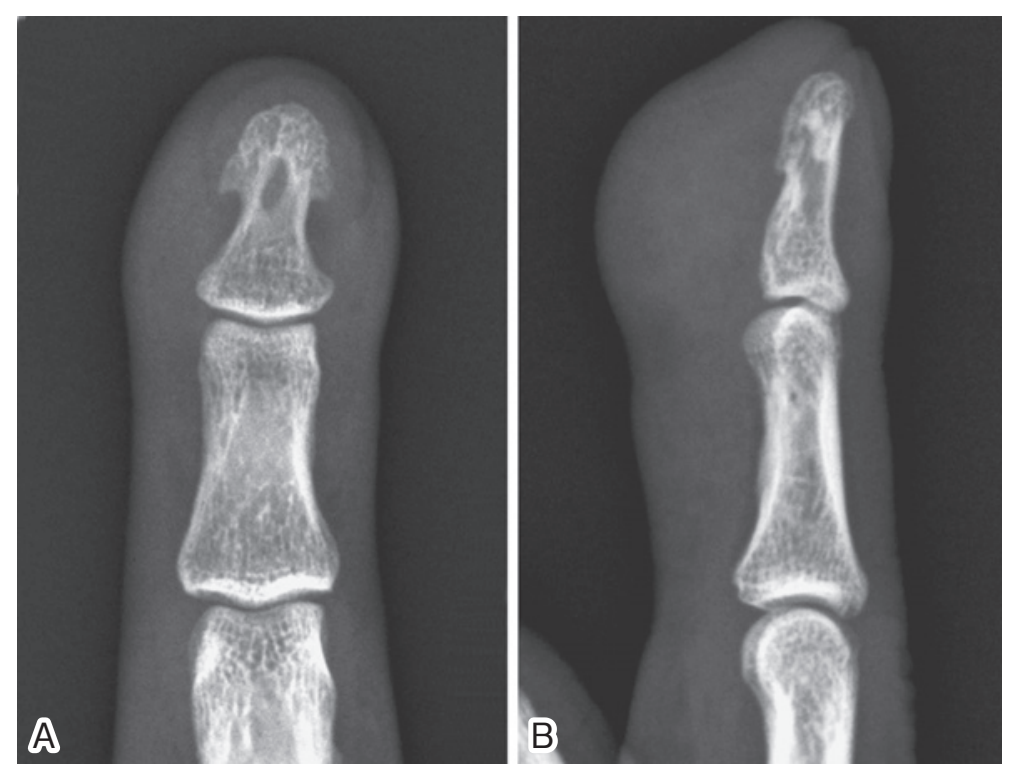

Fig. 2 (A) An anteroposterior radiograph of the index finger. Bone erosion at the slightly distal part of the center of the distal phalanx. (B) A lateral radiograph. Bone erosion extending into the medullary space. 


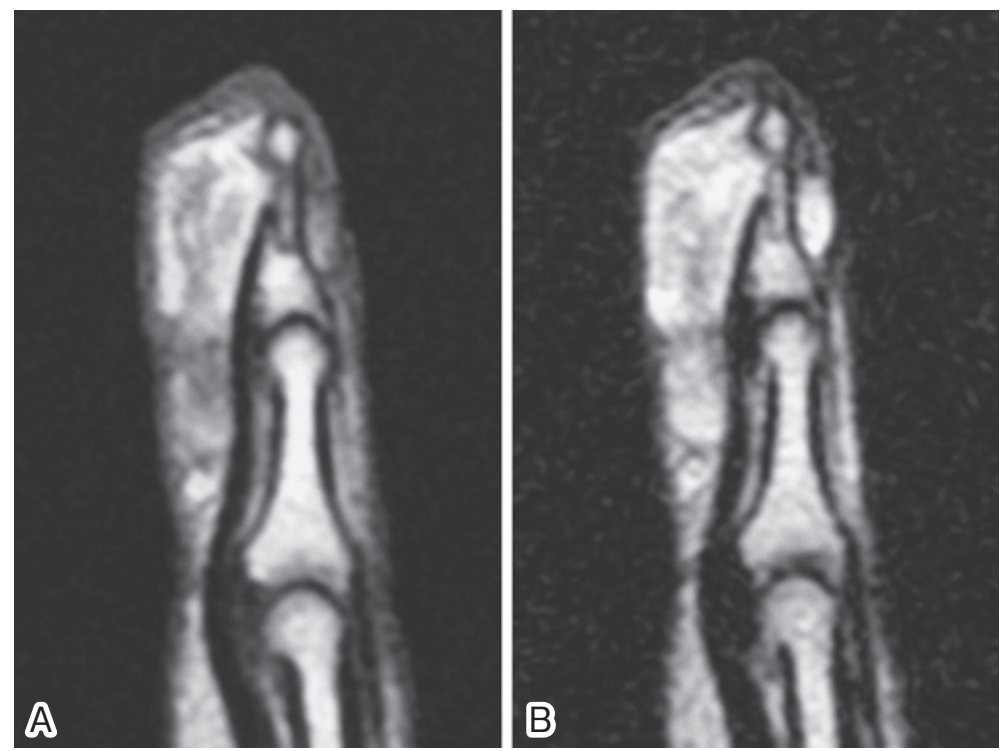

Fig. 3 A T1-weighted image (A) and a T2-weighted image (B) of a sagittal view of the index finger. A soft tissue mass showing high signal intensities with slight irregularity on both T1- and T2-weighted images.

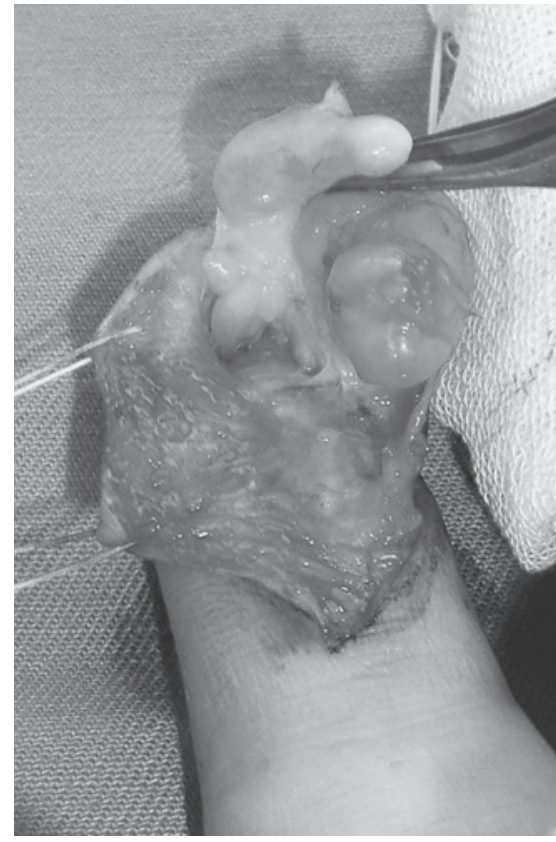

Fig. 4 A yellow, soft, lobulated lesion extending into the bone.

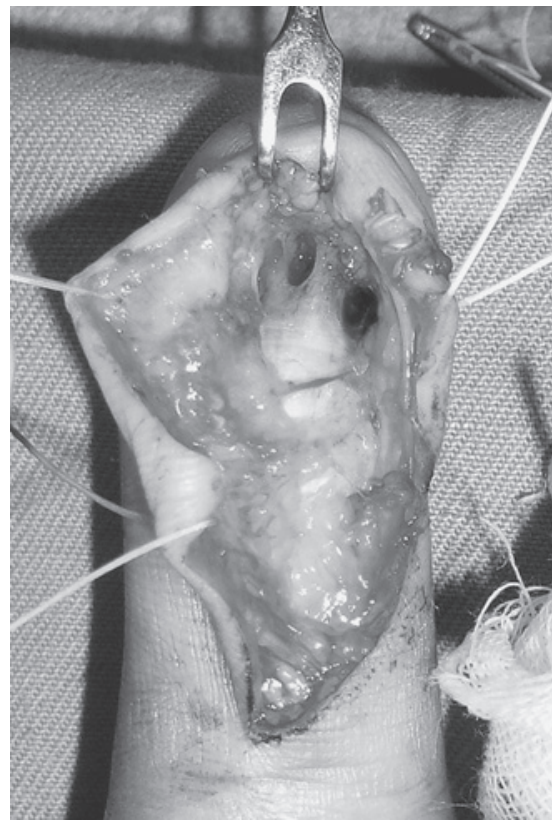

Fig. 5 Deep erosion on the palmar aspect of the distal phalanx. the dorsal side along the radial surface of the distal phalanx (Fig. 4) and was partially adherent to the periosteum. The lesion was easily excised because it did not adhere to the surface of the eroded area. The lesion measured $31 \times 26 \times 12 \mathrm{~mm}$. A deep bone erosion was observed on the palmar aspect of the distal phalanx (Fig. 5). Hyperostotic changes, such as osseous projection, were not found. The diagnosis of lipoma was confirmed with postoperative histological examination (Fig. 6). This examination showed that fibrous tissues were slightly more prevalent than in a typical lipoma. No atypical lipoblasts were observed. The pain resolved after surgery. There was no recurrence during the 1 year of follow-up. 


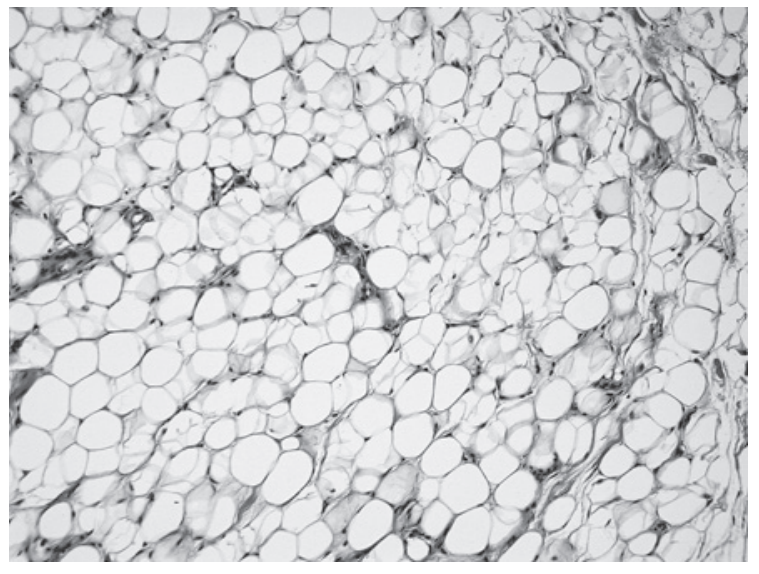

Fig. 6 Histological examination of the lesion showing mature fat cells (H \& E stain, $\times$ 200).

\section{Discussion}

Lipoma of the finger is rare. To the best of our knowledge, fewer than 30 cases have been reported $^{1-13}$. According to detailed reports of 13 lesions, the mean age of the patients was 54; females were slightly predominant; almost all patients had symptoms, such as pain and restricted range of motion; the mean interval between tumor awareness and consultation was 90 months; and tumors did not recur ${ }^{1-13}$.

To the best of our knowledge, only 3 lipomas of the finger associated with bone changes have been reported $^{1-3}$. These were a lipoma causing bone erosion and 2 parosteal lipomas ${ }^{1-3}$. Stein ${ }^{2}$ reported on a 70-year-old man who had a large lump that filled most of the pulp of the thumb. Radiographic images showed a destructive lesion of the distal phalanx. At surgery, there was a small opening into the distal phalanx and multiple loculated areas in the phalanx similar to a honeycomb stone. The lesion was histologically diagnosed as a lipoma. Stein ${ }^{2}$ could not determine whether this lipoma had arisen arose from the pulp of the thumb and invaded the bone or had arisen from the bone and invaded the soft tissues. Therefore, intraosseous lipoma that had invaded the soft tissue could also have been included in the differential diagnosis of the present case. In general, intraosseous lipoma is radiologically visualized as a lytic lesion with trabeculation and may be accompanied by bone expansion and cortical thinning ${ }^{14}$. The present lesion did not show trabeculation, bone expansion, or cortical thinning. In addition, the intraosseous portion of the present lesion was much smaller than the soft-tissue portion. The bone changes associated with the present lesion are not consistent with those associated with intraosseous lipoma.

Parosteal lipoma is a lipoma arising on the bone surface $^{3,15}$. This tumor usually has a broad-based attachment to the subjacent bone and no continuity with the medullary cavities ${ }^{3,15}$. Parosteal lipoma is also usually associated with irregular osseous excrescence into the soft tissue. One of the 2 reported cases was in a 68-year-old woman with a parosteal lipoma at the tip of the fifth finger ${ }^{3}$. Radiographic images showed an osseous projection, cortical irregularity, and hyperostosis of the distal phalan $x^{3}$. These findings were typical of parosteal lipoma. The other case was in a 64-year-old man with a parosteal lipoma of the index finger. This lesion had caused a subtle erosion of the cortex on radiographic images ${ }^{4}$. Regarding the lesion of the present case, its attachment to the phalanx was narrow-based, its extension into the medullary cavities was deep, and it had no association with irregular osseous excrescence. These findings are not consistent with parosteal lipoma. This lesion seemed to be under a high internal pressure, as indicated by the tense skin. The partial adherence of the lesion to the periosteum observed at surgery could be associated with either a parosteal lipoma or a large lipoma. We believe that the present lesion was a long-standing lipoma that had caused deep bone erosion in the finger where the space is limited for tumor growth. Lipoma should be included in the differential diagnosis of a finger tumor, even when bone erosion is seen on radiographs.

\section{References}

1. Brooks ML, Mayer DP, Grannick MS, Solomon MP, Rhoda CH: Parosteal lipoma of the finger: preoperative evaluation with computed tomography. Comput Med Imaging Graph 1989; 13: 481-485.

2. Stein AH Jr: Benign neoplastic and nonneoplastic destructive lesions in the long bones of the hand. Surg Gynecol Obstet 1959; 109: 189-197. 
3. Yamamoto T, Marui T, Mizuno K: Parosteal lipoma of the distal phalanx: a case report and review of the literature. Clin Orthop 2001; 389: 181-184.

4. Baran R: Periungual lipoma at an unusual site. J Dermatol Surg Oncol 1984; 10: 32-33.

5. Bardazzi F, Savoia F, Fanti PA: Subungual lipoma. Br J Dermatol 2003; 149: 418

6. Choi HS, Yi JH, Kang SH, Yun SK: Lipoma of the index finger. J Dermatol 2003; 30: 834-836.

7. Copcu E: Posttraumatic fingertip lipoma. Plast Reconstr Surg 2004; 114: 1986-1987.

8. de Giorgi V, Salvini C, Sestini S, Alfaioli B, Carli P: Lipoma of the finger: a case report and differential diagnosis. Clin Exp Dermatol 2005; 30: 439-440.

9. Failla JM: Subungual lipoma, squamous carcinoma of the nail bed, and secondary chronic infection. J Hand Surg [Am] 1996; 21: 512-514.

10. Hussain M, Riordan C: Lipoma of the finger: a case report. Acta Chir Plast 2003; 45: 16-17.
11. Kalisman M, Beck AR: Lipoma of the thumb in a child. Ann Plast Surg 1979; 2: 165-166.

12. Richert B, André J, Choffray A, Rahier S, de la Brassinne M: Periungual lipoma: about three cases. J Am Acad Dermatol 2004; 51: S91-93.

13. Stuffer M, Thurner JE: Lipoma of the index digit: a very rare location. Arch Orthop Trauma Surg 1995; 114: 239-240.

14. Dorfman HD, Czerniak B: Bone tumors, 1998; pp 913-918, Mosby, St. Louis.

15. Nielsen GP, Mandahl N: Lipoma. In WHO classification of tumours: tumours of soft tissue and bone (Fletcher CD, Unni KK, Mertens F, eds), 2002; pp 20-22, IARC Press, Lyon.

(Received, October 28, 2011)

(Accepted, December 15, 2011) 